\title{
Structural rearrangements of Ru nanoparticles supported on carbon nanotubes under microwave irradiation $\dagger$
}

\author{
Bingsen Zhang, ${ }^{a}$ Xiaojuan Ni, ${ }^{b}$ Wei Zhang, ${ }^{a}$ Lidong Shao, ${ }^{a}$ Qiang Zhang, ${ }^{a}$ Frank Girgsdies, ${ }^{a}$ \\ Changhai Liang, ${ }^{b}$ Robert Schlögl ${ }^{a}$ and Dang Sheng Su*ac
}

Received 28th June 2011, Accepted 15th August 2011

DOI: $10.1039 / \mathrm{clcc13858h}$

The structure evolution of twinned Ru nanoparticles supported on carbon nanotubes rearranging into $\mathrm{Ru}$ single nanocrystals under the microwave irradiation and the exposed surface of $R u$ single crystals were observed, which provided new insights into synthesis and application of metal nanoparticle catalysts.

Metal nanoparticles (NPs) exhibiting fascinating chemical, optical, electronic, and magnetic properties that are often radically different from their bulk counterparts have received wide recognition due to their unique applications in catalysis fields. Various catalytic processes based on metal NPs have been developed, e.g., carbon monoxide oxidation over Au NPs, ${ }^{1}$ ammonia decomposition over $\mathrm{Ru} \mathrm{NPs},{ }^{2}$ and carbon nanotube (CNT) growth on $\mathrm{Fe}$ NPs. ${ }^{3}$ The metal NPs were usually distributed on various supports, such as $\mathrm{Al}_{2} \mathrm{O}_{3}, \mathrm{SiO}_{2}$, carbon black, CNTs, to obtain good dispersion and large exposed surfaces. Not only the electronic structure of the support but also the possible interaction with the support being unavoidable in chemical preparation routes exhibit strong stabilizing effects on $\mathrm{NPs},{ }^{4}$ which are directly associated with the structure of NPs. The fine 3D structure terminated surface, facet edges and corners of NPs always play a key role in the heterogeneous catalysis process. ${ }^{2 g, 5}$ However, the detailed fine structures of ultra-small NPs were less resolved due to the poor image contrast of the NPs on the thick catalyst supports. Since the crystallographic orientation effect on the catalytic reactions was highly concerned, ${ }^{2 g, 5}$ the first step is to identify the real structure and exposed surfaces of the ultra-small metal NP catalyst.

Modern Cs-corrected high-resolution transmission electron microscopy (HRTEM) techniques can be used to complement scanning probe and surface diffraction methods for revealing

${ }^{a}$ Department of Inorganic Chemistry, Fritz Haber Institute of the Max Planck Society, Faradayweg 4-6, 14195 Berlin, Germany.

E-mail: dangsheng@fhi-berlin.mpg.de; Fax: + 493084134401

${ }^{b}$ Laboratory of Advanced Materials and Catalytic Engineering, School of Chemical Engineering, Dalian University of Technology, 2 Linggong Road, 116012 Dalian, China

${ }^{c}$ Shenyang National Laboratory for Materials Science, Institute of Metal Research, Chinese Academy of Science, 72 Wenhua Road, 110006 Shenyang, China

$\dagger$ Electronic supplementary information (ESI) available: Experimental details, HRTEM images and corresponding shapes, the frequency of single crystal Ru NPs against particle size for each sample, and particle size distribution statistical data. See DOI: $10.1039 / \mathrm{clcc} 13858 \mathrm{~h}$ the local and surface structure of catalysts. ${ }^{6}$ The next step is to prepare ultra-small NP samples visible for TEM, for instance, dispersed metal NPs on an ultra-thin nanocarbon (CNTs and graphene). The $\mathrm{Ru} / \mathrm{CNT}$ s based catalysts, which were critical for fuel cells, ${ }^{7}$ cellulose conversion into sorbitol, ${ }^{8}$ ammonia synthesis ${ }^{9}$ and decomposition, ${ }^{2 a-c}$ hydrogenation, ${ }^{10}$ and selective oxidation, ${ }^{11}$ were selected as model catalysts. They were prepared by the microwave thermolysis method that is suitable for preparation of nanomaterials with the advantages of rapid reaction rate, uniform heating, and energy saving. ${ }^{12}$ The Ru/CNTs were characterized by a FEI Titan 80-300 Cs-corrected electron microscope in this communication. Their structure evolution under the microwave irradiation and exposed surface were investigated to reveal more crystallographic features of a real Ru NP.

Briefly, the $5.0 \mathrm{wt} \% \mathrm{Ru} / \mathrm{CNT}$ s catalysts were synthesized by a microwave thermolysis method. Firstly, the $\mathrm{Ru}_{3}(\mathrm{CO})_{12}$ was mixed together with CNTs and grinded; then the mixture was kept in Ar flow for $2 \mathrm{~h}$, followed by microwave treatment at a quartz-tube reactor with a frequency of $2.45 \mathrm{GHz}$ (the power is $800 \mathrm{~W}$ ) as the radiation source for 1,3 , and $5 \mathrm{~min}$. The $\mathrm{Ru} /$ CNTs catalysts were then taken out after cooling to room temperature, and named as $\mathrm{Ru} / \mathrm{CNTs}-1 \mathrm{~min}, \mathrm{Ru} / \mathrm{CNTs}-3 \mathrm{~min}$, and $\mathrm{Ru} / \mathrm{CNTs}-5 \mathrm{~min}$, respectively. Fig. 1 shows the X-ray diffraction (XRD) patterns of CNTs and Ru/CNTs. Besides diffraction peaks of CNTs as supports, the characteristic diffraction peaks of Ru particles (100), (101), and (110) can be indexed. While the XRD pattern of CNTs maintains,

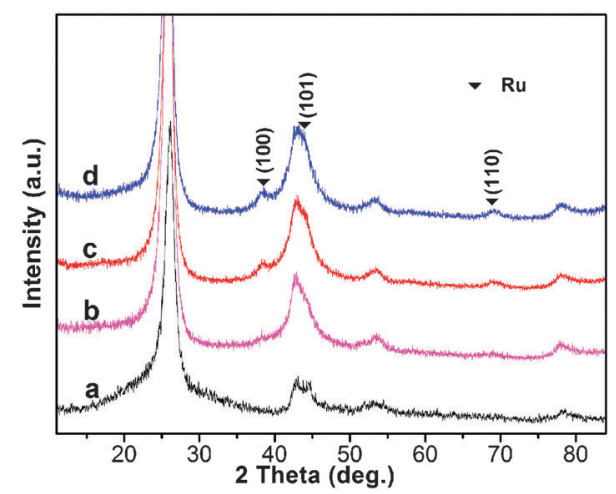

Fig. 1 XRD patterns of CNTs (a), Ru/CNTs-1 min (b), Ru/CNTs-3 min (c), and $\mathrm{Ru} / \mathrm{CNTs}-5$ min (d) samples. 

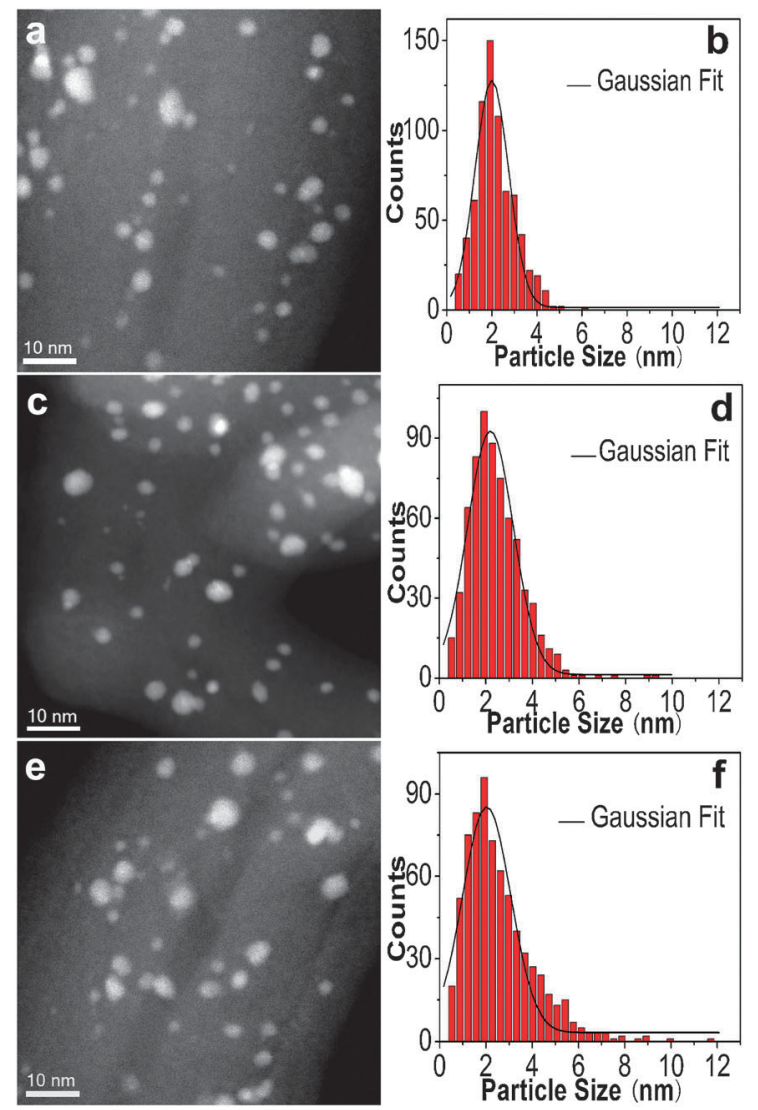

Fig. 2 STEM (a, c, e) and the corresponding histograms of PSD (b, d, f) images of Ru/CNTs- 1 min, Ru/CNTs-3 min, and Ru/CNTs- 5 min samples.

an obvious evolution occurred for the diffraction peaks of $\mathrm{Ru}$. The intensities of (100) and (110) peaks of Ru increase with the irradiation time, which indicates an increase of crystallinity of $\mathrm{Ru}$ particles. Although the $\mathrm{Ru}$ (101) is partially overlapped with the peak of CNTs, the aforementioned increase of intensity can be tracked here. Fig. 2 displays typical scanning transmission electron microscopy (STEM) images of Ru/CNT samples together with the histograms of particle size distribution (PSD) with a good accuracy. ${ }^{13}$ An increase of the mean value of $\mathrm{Ru}$ particle size was observed from 1.8 via 2.3 to $2.6 \mathrm{~nm}$ with the microwave irradiation times 1,3 and $5 \mathrm{~min}$, but with a narrow range (Fig. 2 and Table $\mathrm{S} 1$, ESI $\dagger$ ).

To reveal the fine structure of Ru NPs on CNTs, HRTEM observations have been conducted (Fig. 3). Many multiply twinned $\mathrm{Ru}$ particles which consist of several subunits were found in the samples under the microwave irradiation for both 1 min (Fig. 3a) and 3 min (Fig. 3b). The Ru particles have been transformed into single crystals with the disappearance of twins after a $5 \mathrm{~min}$ irradiation. As shown in Fig. 3c, the lattice fringes (10-1) and (1-10) of Ru with a characteristic acute angle of $63.9^{\circ}$ were identified on a typical NP based on the PDF card (No. 06-0663) with space group P63/mmc. The HRTEM image and the simulated [111] projected particle image with faceting configurations match well with each other (see the inset of Fig. 3c). Fig. S1a and c (ESI $\dagger$ ) show the HRTEM images of different single crystal Ru particles in the Ru/CNTs$5 \mathrm{~min}$. The exposed facets of these three Ru particles (Fig. 3c; Sla and c, ESI $\dagger$ ) supported onto CNTs can be identified as

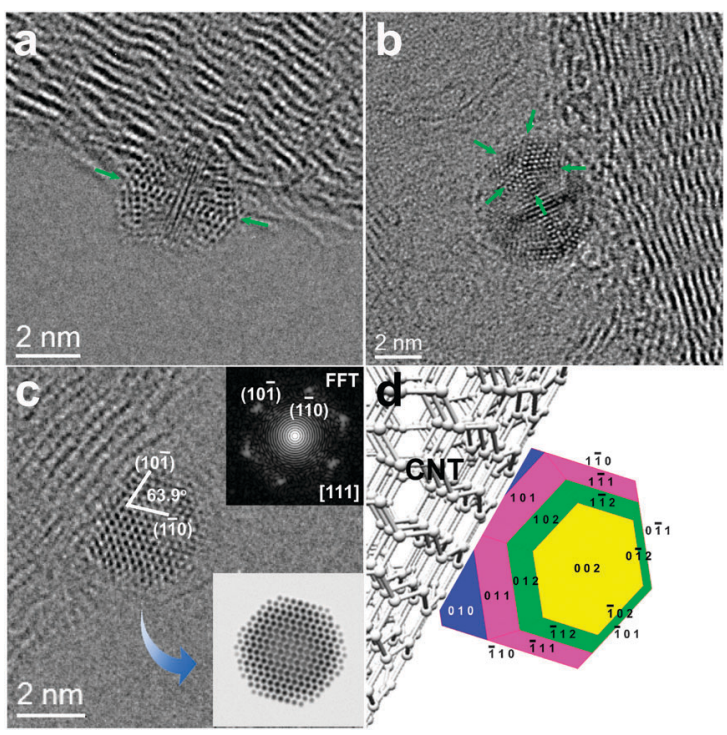

Fig. 3 Typical HRTEM images of Ru/CNTs-1 min (a), Ru/CNTs-3 min (b), and Ru/CNTs-5 min (c) samples. The corresponding shape with faceting configurations of (c) (d). Insets in (c) are the local Fast Fourier Transform (FFT) of the HRTEM image and a simulated HRTEM image well matched its experimental counterpart.

$\{012\},\{112\},\{111\},\{100\},\{101\}$, and $\{002\}$ planes (Fig. 3d; S1b and $d$, ESI $\dagger$ ). On the basis of the Wulff construction, ${ }^{14}$ the exposed facets of most Ru particles are approximately terminated by these planes mentioned above, on which there exists some surface roughness, ascribed to the migration of surface atoms, forming corners and steps. Similarly, the facets of $\mathrm{Ru}$ particles in contact with CNTs can also be identified, e.g., (10-1) (see Fig. 3c).

Furthermore, in order to explore the structure evolution under microwave irradiation, the ratio of single $\mathrm{Ru}$ crystals to all particles (Fig. 4d) and the frequency of single crystal Ru NPs against particle size for each sample (Fig. S2, ESI $\dagger$ ) were measured. As shown by the white circles in Fig. 4a-c, the single crystal $\mathrm{Ru}$ particles can be distinguished from multiply twinned particles (MTPs). The ratio of single crystals amongst all particles was obtained through an examination of more than 300 random particles for each sample. Fig. 4 d reveals that the ratios are $24 \%, 49 \%$, and $92 \%$ for $\mathrm{Ru} / \mathrm{CNTs}-1 \mathrm{~min}, 3 \mathrm{~min}$, and $5 \mathrm{~min}$, respectively. The crystallinity of $\mathrm{Ru} / \mathrm{CNTs}-5 \mathrm{~min}$ is better than that of other samples. On the other hand, the frequency of single crystal NPs against particle size (Fig. S2, ESI $\dagger$ ) indicates that the single crystal formation is mainly due to the microwave irradiation. During the microwave irradiation process, the metal or conductive object placed in the microwave will act as an antenna to some degree, resulting in an electric current, which acts as a heating element. ${ }^{15}$ Both the $\mathrm{Ru}$ NPs and CNTs absorb the microwave energy. The high thermal and electronic conductivity of CNTs render the catalysts a uniform volumetric heating at a high heating rate. Thus, the formation of MTPs is favored by extensive faceting, small twin boundary energies, and small or negative surface stresses, which is similar to that of twined crystal growth during hydrothermal processing. ${ }^{16}$ With the increase of the microwave energy input, the migration of the twin boundary could result in a single crystal, which is a more thermodynamic stable form of $\mathrm{Ru}$ NPs. The single crystal $\mathrm{Ru}$ particle was 


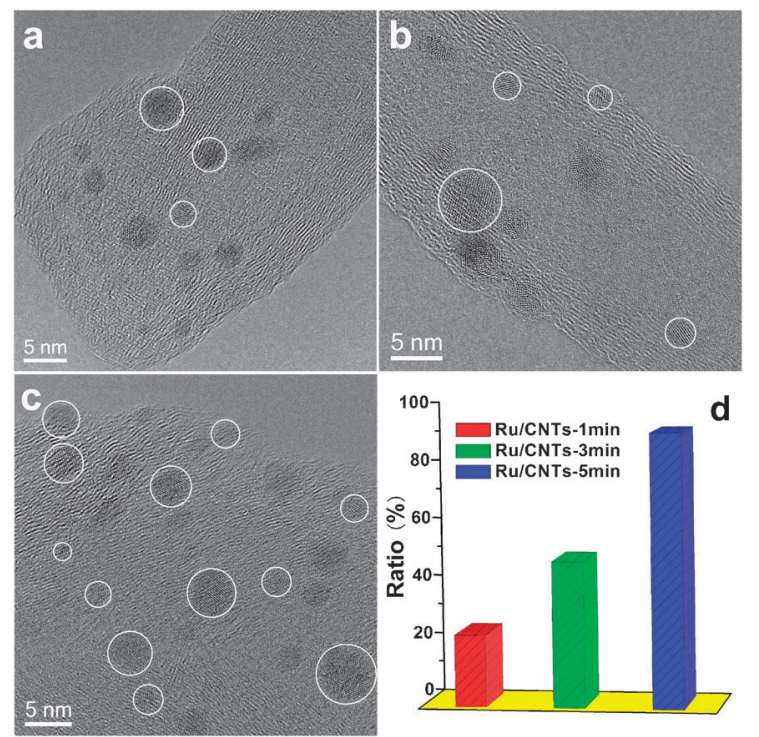

Fig. 4 HRTEM images of Ru/CNTs-1 min (a), Ru/CNTs-3 min (b), and $\mathrm{Ru} / \mathrm{CNTs}-5$ min (c) samples. (White circles are referred to as the single crystal particles in a-c.). (d) The histogram for the ratio of the single crystal particle number to all of the particle numbers.

achieved through the possible preferred growth of some particular planes, e.g., the growth of $\mathrm{Ru}(001)$ can facilitate detwinning of $\mathrm{Ru} .{ }^{17}$ Thus, successive structural rearrangement of MTPs near grain boundaries leads to the annihilation of the twins. Excess heat will tend to drive ripening processes, which leads to the size of Ru NPs becoming larger under long time microwave irradiation. The high surface area and onedimensional hollow structures of CNTs have been utilized in supporting metal species for heterogeneous catalysis. Microwave-thermolysis allows a rapid formation of NPs on CNTs without further thermal treatments. Moreover, current synthesis takes advantages of the interactions between metal atoms and localized double bonds on carbon nanostructures with curved basic structural units, ${ }^{18}$ thus, Ru NPs can be nucleated and uniformly anchored on the CNT surface and structure rearrangement can be investigated in detail.

In summary, the structural rearrangement of Ru NPs and their exposed surfaces were identified by Cs-corrected electron microscopy. The structure evolution of Ru from MTPs to single crystals under microwave irradiation was unambiguously identified. The as-obtained Ru NPs have an interaction with the CNTs support and a narrow size distribution. The ratio of Ru single crystals to all NPs can be increased to $92 \%$ when the irradiation was carried out for $5 \mathrm{~min}$. These results suggest a new pathway of crystallization of metal NPs on CNTs. The simple fabrication art may benefit applications utilizing Ru single crystal NPs, especially in cases of supported $\mathrm{Ru}$ catalysts with metal particles in the nanoscale. Microscopy studies in the present work provide a good modelling system on structural evolution of metal NPs with different stages.

The authors thank Edith Kitzelmann for her help with XRD characterization.

\section{Notes and references}

1 (a) A. S. K. Hashmi and G. J. Hutchings, Angew. Chem., Int. Ed., 2006, 45, 7896; (b) K. F. Zhao, B. T. Qiao, J. H. Wang, Y. J. Zhang and T. Zhang, Chem. Commun., 2011, 47, 1779.
2 (a) W. Q. Zheng, J. Zhang, B. Zhu, R. Blume, Y. L. Zhang, K. Schlichte, R. Schlogl, F. Schuth and D. S. Su, ChemSusChem, 2010, 3, 226; (b) F. R. Garcia-Garcia, J. Alvarez-Rodriguez, I. Rodriguez-Ramos and A. Guerrero-Ruiz, Carbon, 2010, 48, 267; (c) J. L. Chen, Z. H. Zhu, S. B. Wang, Q. Ma, V. Rudolph and G. Q. Lu, Chem. Eng. J., 2010, 156, 404; (d) Y. X. Li, L. H. Yao, Y. Y. Song, S. Q. Liu, J. Zhao, W. J. Ji and C. T. Au, Chem. Commun., 2010, 46, 5298; (e) X. Z. Duan, J. H. Zhou, G. Qian, P. Li, X. G. Zhou and D. Chen, Chin. J. Catal., 2010, 31, 979; (f) B. Lorenzut, T. Montini, C. C. Pavel, M. Comotti, F. Vizza, C. Bianchini and P. Fornasiero, ChemCatChem, 2010, 2, 1096; (g) A. M. Karim, V. Prasad, G. Mpourmpakis, W. W. Lonergan, A. I. Frenkel, J. G. G. Chen and D. G. Vlachos, J. Am. Chem. Soc., 2009, 131, 12230.

3 (a) M. Q. Zhao, Q. Zhang, W. Zhang, J. Q. Huang, Y. H. Zhang, D. S. Su and F. Wei, J. Am. Chem. Soc., 2010, 132, 14739; (b) M. Q. Zhao, Q. Zhang, J. Q. Huang, J. Q. Nie and F. Wei, Carbon, 2010, 48, 3260; (c) M. Q. Zhao, Q. Zhang, X. L. Jia, J. Q. Huang, Y. H. Zhang and F. Wei, Adv. Funct. Mater., 2010, 20, 677; (d) J. Q. Huang, Q. Zhang, M. Q. Zhao, G. H. Xu and F. Wei, Nanoscale, 2010, 2, 1401.

4 M. A. Brown, E. Carrasco, M. Sterrer and H. J. Freund, J. Am. Chem. Soc., 2010, 132, 4064.

5 (a) X. W. Xie, Y. Li, Z. Q. Liu, M. Haruta and W. J. Shen, Nature, 2009, 458, 746; (b) L. D. Shao, W. Zhang, M. Armbrüster, D. Teschner, F. Girgsdies, B. S. Zhang, O. Timpe, M. Friedrich, R. Schlögl and D. S. Su, Angew. Chem., Int. Ed., 2011, 50, DOI: 10.1002/anie.201008013.

6 (a) D. S. Su, T. Jacob, T. W. Hansen, D. Wang, R. Schlogl, B. Freitag and S. Kujawa, Angew. Chem., Int. Ed., 2008, 47, 5005; (b) W. Zhang, A. Trunschke, R. Schlogl and D. S. Su, Angew. Chem., Int. Ed., 2010, 49, 6084; (c) D. S. Su, Angew. Chem., Int. Ed., 2010, 49, 9569; (d) T. Ling, L. Xie, J. Zhu, H. M. Yu, H. Q. Ye, R. Yu, Z. Cheng, L. Liu, G. W. Yang, Z. D. Cheng, Y. J. Wang and X. L. Ma, Nano Lett., 2009, 9, 1572.

7 (a) R. Chetty, S. Kundu, W. Xia, M. Bron, W. Schuhmann, V. Chirila, W. Brandl, T. Reinecke and M. Muhler, Electrochim. Acta, 2009, 54, 4208; (b) R. T. Lv, T. X. Cui, M. S. Jun, Q. A. Zhang, A. Y. Cao, D. S. Su, Z. J. Zhang, S. H. Yoon, J. Miyawaki, I. Mochida and F. Y. Kang, Adv. Funct. Mater., 2011, 21, 999.

8 W. P. Deng, X. S. Tan, W. H. Fang, Q. H. Zhang and Y. Wang, Catal. Lett., 2009, 133, 167.

9 S. J. Guo, X. L. Pan, H. L. Gao, Z. Q. Yang, J. J. Zhao and X. H. Bao, Chem.-Eur. J., 2010, 16, 5379.

10 Z. Y. Sun, Z. M. Liu, B. X. Han, Y. Wang, J. M. Du, Z. L. Xie and G. J. Han, Adv. Mater., 2005, 17, 928.

11 X. M. Yang, X. N. Wang and J. S. Qiu, Appl. Catal., A, 2010, 382, 131.

12 (a) C. H. Liang, L. Ding, C. A. Li, M. Pang, D. S. Su, W. Z. Li and Y. M. Wang, Energy Environ. Sci., 2010, 3, 1121; (b) C. H. Liang, L. Ding, A. Q. Wang, Z. Q. Ma, J. S. Qiu and T. Zhang, Ind. Eng. Chem. Res., 2009, 48, 3244; (c) M. Pang, C. Li, L. Ding, J. Zhang, D. S. Su, W. Z. Li and C. H. Liang, Ind. Eng. Chem. Res., 2010, 49, 4169

13 B. S. Zhang, W. Zhang and D. S. Su, Chem CatChem, 2011, 3, 965.

14 (a) G. Wulff, Z. Kristallogr., 1901, 34, 449; (b) K. H. Hansen, T. Worren, S. Stempel, E. Laegsgaard, M. Baumer, H. J. Freund, F. Besenbacher and I. Stensgaard, Phys. Rev. Lett., 1999, 83, 4120; (c) P. L. Hansen, J. B. Wagner, S. Helveg, J. R. Rostrup-Nielsen, B. S. Clausen and H. Topsoe, Science, 2002, 295, 2053.

15 J. A. Dahl, B. L. S. Maddux and J. E. Hutchison, Chem. Rev., 2007, 107, 2228

16 (a) W. C. Zhu, Q. Zhang, L. Xiang, F. Wei, X. T. Sun, X. L. Piao and S. L. Zhu, Cryst. Growth Des., 2008, 8, 2938; (b) X. L. Hu, Y. Masuda, T. Ohji and K. Kato, Cryst. Growth Des., 2009, 9, 3598; (c) W. C. Zhu, S. L. Zhu and L. Xiang, CrystEngComm, 2009, 11, 1910.

17 W. L. Ling, N. C. Bartelt, K. F. McCarty and C. B. Carter, Phys. Rev. Lett., 2005, 95, 166105.

18 (a) J. P. Tessonnier, D. Rosenthal, F. Girgsdies, J. Amadou, D. Begin, C. Pham-Huu, D. S. Su and R. Schlogl, Chem. Commun., 2009, 7158; (b) E. P. J. Parrott, J. A. Zeitler, J. McGregor, S. P. Oei, H. E. Unalan, S. C. Tan, W. I. Milne, J. P. Tessonnier, R. Schlogl and L. F. Gladden, J. Phys. Chem. C, 2009, 113, 10554 . 